\title{
Mixed-integer non-linear programming approach to structural optimization
}

\author{
S. Kravanja \\ University of Maribor, Faculty of Civil Engineering, Maribor, Slovenia
}

\begin{abstract}
The paper presents the Mixed-Integer Non-Linear Programming (MINLP) approach to structural optimization. MINLP is a combined discrete/continuous optimization technique, where discrete binary 0-1 variables are defined for optimization of discrete alternatives and continuous variables for optimization of parameters. The MINLP optimization is performed through three steps: i.e. the generation of a mechanical superstructure, the modelling of an MINLP model formulation and the solution of the defined MINLP problem. As the discrete/continuous optimization problems are usually non-convex and highly non-linear, the Modified Outer-Approximation/Equality-Relaxation (OA/ER) algorithm is applied for the optimization. The accompanied Linked Multilevel Hierarchical Strategy (LMHS) is developed to accelerate the convergence of the mentioned algorithm. Two examples are presented at the end of the paper.
\end{abstract}

Keywords: mixed-integer non-linear programming, MINLP, structural optimization, structural synthesis.

\section{Introduction}

The paper presents the Mixed-Integer Non-Linear Programming (MINLP) approach to structural optimization. MINLP handles continuous and discrete binary 0-1 variables simultaneously. While continuous variables are defined for the continuous optimization of parameters (dimensions, stresses, strains, weights, costs, etc.), discrete variables are used to express discrete decisions, i.e. usually the existence or non-existence of structural elements inside the defined structure. Different materials, standard dimensions and rounded dimensions may also be defined as discrete alternatives. Since continuous and discrete optimizations are carried out simultaneously, the MINLP approach also finds optimal continuous 
parameters, structural topology, material, standard and rounded dimensions simultaneously.

The MINLP discrete/continuous optimization problems of structural optimization are in most cases comprehensive, non-convex and highly nonlinear. The MINLP optimization approach is proposed to be performed through three steps:

- Generation of MINLP superstructure for different structural/topology and other design alternatives that are candidates for a feasible and optimal solution.

- Development of a special MINLP model formulation for the defined superstructure in an equation oriented environment.

- Solution of the defined MINLP model, performed by suitable MINLP algorithm and strategies, which in the simultaneous MINLP optimization approach yield an optimal structural topology, material, shape, standard cross-sections and rounded dimensions.

Many different methods for solving MINLP problems have been developed in the near past. This paper reports the experience in solving the optimization problems by using the Outer-Approximation/Equality-Relaxation (OA/ER) algorithm [1, 2]. The Linked Multilevel Hierarchical strategy (LMHS) has been developed to accelerate the convergence of the mentioned algorithm. Since the number of discrete alternatives and defined binary 0-1 variables are usually too high for normal solution of the MINLP, a special reduction procedure is developed to reduce automatically the number of binary variables on a reasonable level.

Two examples are presented at the end of the paper. The first example shows the material and standard dimension optimization of a $32 \mathrm{~m}$ long composite I beam floor system and the second example deals with the simultaneous topology, material and standard section optimization of a single-storey industrial steel building structure.

\section{Mechanical superstructure}

The MINLP optimization approach to structural optimization requires the generation of an MINLP mechanical superstructure composed of various topology and design alternatives that are all candidates for a feasible and optimal solution. While topology alternatives represent different selections and interconnections of corresponding structural elements, design alternatives include different materials, standard and rounded dimensions.

The superstructure is typically described by means of unit representation: i.e. structural elements and their interconnection nodes. Each potential topology alternative is represented by a special number and a configuration of selected structural elements and their interconnections; each structural element may in addition have different material, standard and rounded dimension alternatives. 
Therefore, the main goal is to find within the given superstructure a feasible structure that is optimal with respect to topology, material, standard and rounded dimensions as well as all defined continuous parameters.

\section{MINLP model formulation for mechanical superstructures}

It is assumed that a general non-convex and non-linear discrete/continuous optimization problem can be formulated as an MINLP problem (MINLP-G) in the form:

$$
\begin{gathered}
\min \quad z=\boldsymbol{c}^{T} \boldsymbol{y}+f(\boldsymbol{x}) \\
\text { s.t. } \quad \boldsymbol{h}(\boldsymbol{x})=\boldsymbol{0} \\
\boldsymbol{g}(\boldsymbol{x}) \leq \boldsymbol{0} \\
\boldsymbol{B} \boldsymbol{y}+\boldsymbol{C} \boldsymbol{x} \leq \boldsymbol{b} \\
\boldsymbol{x} \in X=\left\{\boldsymbol{x} \in R^{n}: \boldsymbol{x}^{\mathrm{LO}} \leq \boldsymbol{x} \leq \boldsymbol{x}^{\mathrm{UP}}\right\} \\
\boldsymbol{y} \in Y=\{0,1\}^{m}
\end{gathered}
$$

where $\boldsymbol{x}$ is a vector of continuous variables specified in the compact set $X$ and $\boldsymbol{y}$ is a vector of discrete, mostly binary $0-1$ variables. Functions $f(\boldsymbol{x}), \boldsymbol{h}(\boldsymbol{x})$ and $\boldsymbol{g}(\boldsymbol{x})$ are non-linear functions involved in the objective function $z$, equality and inequality constraints, respectively. Finally, $\boldsymbol{B} \boldsymbol{y}+\boldsymbol{C} \boldsymbol{x} \leq \boldsymbol{b}$ represents a subset of mixed linear equality/inequality constraints.

The above general MINLP model formulation has been adapted for the optimization of mechanical superstructures (MINLP-MS). The resulted formulation is more specific, particularly in variables and constraints. It can be used also for the modelling of any mechanical structure. It is given in the following form:

$$
\begin{gathered}
\min \quad z=\boldsymbol{c}^{T} \boldsymbol{y}+f(\boldsymbol{x}) \\
\text { s.t. } \quad \boldsymbol{h}(\boldsymbol{x})=\boldsymbol{0} \\
\boldsymbol{g}(\boldsymbol{x}) \leq \boldsymbol{0} \\
\boldsymbol{A}(\boldsymbol{x}) \leq \boldsymbol{a} \\
\boldsymbol{E} \boldsymbol{y} \leq \boldsymbol{e} \\
\boldsymbol{y}^{e}+\boldsymbol{R}(\boldsymbol{x}) \leq \boldsymbol{r} \\
\boldsymbol{K} \boldsymbol{y}^{e}+\boldsymbol{L}\left(\boldsymbol{d}^{c n}\right) \leq \boldsymbol{k} \\
\boldsymbol{P} \boldsymbol{y}+\boldsymbol{S}\left(\boldsymbol{d}^{m}\right) \leq \boldsymbol{s} \\
\boldsymbol{M y}+\boldsymbol{N}\left(\boldsymbol{d}^{s t}\right) \leq \boldsymbol{t} \\
\boldsymbol{x} \in X=\left\{\boldsymbol{x} \in R{ }^{n}: \boldsymbol{x}^{\mathrm{LO}} \leq \boldsymbol{x} \leq \boldsymbol{x}^{\mathrm{UP}}\right\} \\
\boldsymbol{y} \in Y=\{0,1\}^{m}
\end{gathered}
$$


The MINLP-MS model formulation for mechanical superstructures is proposed to be described as follows:

- Included are continuous variables $\boldsymbol{x}=\{\boldsymbol{d}, \boldsymbol{p}\}$ and discrete binary variables $\boldsymbol{y}=\left\{\boldsymbol{y}^{e}, \boldsymbol{y}^{m}, \boldsymbol{y}^{s t}\right\}$. Continuous variables are partitioned into design variables $\boldsymbol{d}=\left\{\boldsymbol{d}^{c n}, \boldsymbol{d}^{m}, \boldsymbol{d}^{s t}\right\}$ and into performance (nondesign) variables $\boldsymbol{p}$, where subvectors $\boldsymbol{d}^{c n}, \boldsymbol{d}^{m}$ and $\boldsymbol{d}^{s t}$ stand for continuous dimensions, discrete materials and standard dimensions, respectively. Subvectors of binary variables $\boldsymbol{y}^{e}, \boldsymbol{y}^{m}$ and $\boldsymbol{y}^{\text {st }}$ denote the potential existence of structural elements inside the superstructure (the topology determination) as well as the potential selection of the discrete materials and standard dimension alternatives, respectively.

- The economical (or mass) objective function $z$ involves fixed cost charges in the linear term $\boldsymbol{c}^{\mathrm{T}} \boldsymbol{y}$ and dimension dependant costs in the term $f(\boldsymbol{x})$.

- Parameter non-linear and linear constraints $\boldsymbol{h}(\boldsymbol{x})=\boldsymbol{0}, \boldsymbol{g}(\boldsymbol{x}) \leq \boldsymbol{0}$ and $\boldsymbol{A}(\boldsymbol{x}) \leq \boldsymbol{a}$ represent the rigorous system of the design, loading, stress, deflection, stability, etc. constraints known from the structural analysis.

- Integer linear constraints $\boldsymbol{E} \boldsymbol{y} \leq \boldsymbol{e}$ are proposed to describe relations between binary variables.

- Mixed linear constraints $\boldsymbol{D y}^{e}+\boldsymbol{R}(\boldsymbol{x}) \leq \boldsymbol{r}$ restore interconnection relations between currently selected or existing structural elements (corresponding $y^{e}$ $=1$ ) and cancel relations for currently disappearing or nonexisting elements (corresponding $y^{e}=0$ ).

- Mixed linear constraints $\boldsymbol{K y}^{e}+\boldsymbol{L}\left(\boldsymbol{d}^{c n}\right) \leq \boldsymbol{k}$ are proposed to define the continuous design variables for each existing structural element. The space is defined only when the corresponding structure element exists $\left(y^{e}=1\right)$, otherwise it is empty.

- Mixed linear constraints $\boldsymbol{P} \boldsymbol{y}+\boldsymbol{S}\left(\boldsymbol{d}^{m}\right) \leq \boldsymbol{s}$ define discrete materials $\boldsymbol{d}^{m}$. The disrete materials are defined in a similar way as the standard dimensions.

- Mixed linear constraints $\boldsymbol{M y}+\boldsymbol{N}\left(\boldsymbol{d}^{\mathrm{st}}\right) \leq \boldsymbol{t}$ define standard discrete design variables $\boldsymbol{d}^{s t}$. Each standard dimension $d^{s t}$ is determined as a scalar product between its vector of standard discrete dimension constants $\boldsymbol{q}$ and its vector of binary variables $\boldsymbol{y}^{s t}$, eqn. (1). Only one discrete value can be selected for each standard dimension since the sum of binary variables must be equal one, eqn. (2):

$$
\begin{gathered}
d^{\mathrm{st}}=\sum_{i \in I} q_{i} y_{i}^{\mathrm{st}} \\
\sum_{i \in I} y_{i}^{\mathrm{st}}=1
\end{gathered}
$$

The uper MINLP-MS model formulation for mechanical superstructures is presented in the condensed form. More about MINLP see Kravanja et al. [2-5].

\section{Solving an MINLP problem}

After the MINLP model formulation is developed, the defined MINLP optimization problem is solved by the use of suitable MINLP algorithm and 
strategies. A general MINLP class of optimization problems can be solved in principle by the following algorithms and their extensions:

- the Nonlinear Branch and Bound, NBB, proposed and used by many authors, e.g. Beale [6], Gupta and Ravindran [7];

- the Sequential Linear Discrete Programming method, SLDP, by Olsen and Vanderplaats [8] and Bremicker et al. [9];

- the Extended Cutting Plane method by Westerlund et al. [10];

- the Generalized Benders Decomposition, GBD, by Benders [11], Geoffrion [12];

- the Outer-Approximation /Equality Relaxation algorithm, OA/ER, by Kocis and Grossmann [13];

- the Feasibility Technique by Mawengkang and Murtagh [14]; and

- the LP/NLP based Branch and Bound algorithm by Quesada and Grossmann [15].

\subsection{Modified OA/ER algorithm}

The OA/ER algorithm seems to be one of the most efficient algorithm to solve large-scale MINLP problems, when NLP subproblems are expensive and difficult to solve. The OA/ER algorithm consists of solving an alternative sequence of Non-linear Programming (NLP) optimization subproblems and Mixed-Integer Linear Programming (MILP) master problems. The former corresponds to continuous optimization of parameters for a mechanical structure with fixed topology (and material, standard and rounded dimensions) and yields an upper bound to the objective to be minimized. The latter involves a global approximation to the superstructure of alternatives in which new topology, materials, standard and rounded dimensions are identified so that its lower bound does not exceed the current best upper bound. The search of a convex problem is terminated when the predicted lower bound exceeds the upper bound, otherwise it is terminated when the NLP solution can be improved no more. The convergence is usually achieved in a few MINLP iterations. The OA/ER algorithm guarantees the global optimality of solutions for convex and quasiconvex optimization problems.

The OA/ER algorithm as well as all other MINLP algorithms do not generally guarantee that the solution found is the global optimum. This is due to the presence of non-convex functions in the models that may cut off the global optimum. In order to reduce undesirable effects of nonconvexities, the Modified OA/ER algorithm was proposed by Kravanja and Grossmann [1] by which the following modifications are applied for the master problem: deactivation of linearizations, decomposition and deactivation of the objective function linearization, use of the penalty function, use of the upper bound on the objective function to be minimized as well as a global convexity test and a validation of the outer approximations.

\subsection{Linked multilevel hierarchical strategy, LMHS}

The optimal solution of comprehensive non-convex and non-linear MINLP problem with a high number of discrete decisions is in general very difficult to 
be obtained. For this purpose, the LMHS strategy has been developed to accelerate the convergence of the OA/ER algorithm. Using the LMHS strategy, we decompose the original integer space and original MINLP problem in a hierarchical manner into several subspaces and corresponding MINLP levels. Each time the next MINLP optimization level is performed, the current integer subspace is extended by the next integer subspace and prescreened, while the discrete decisions belonging to all of the remaining subspaces are approximated by the relaxed $0-1$ variables. The levels are linked by accumulating outerapproximations and yield lower bounds to their next level objective functions to be minimized, which considerably improve the efficiency of the search. Decision levels are hierarchically classified as:

- The level of discrete topology and material alternatives (the highest level).

- The level of discrete standard dimension decisions (the middle level).

- The level of rounded continuous dimension decisions (the lower level).

Higher levels give lower bounds to the original objective function to be minimized while lower levels give upper bounds. The MINLP subproblems are iterated about each level until there are no improvements in the NLP solution. Thus, we start with the discrete topology and material optimization at the relaxed standard dimensions. When the optimal topology and materials are reached, we proceed with simultaneous discrete topology, material and standard dimension optimization at the second level. Finally, after the optimal topology, materials and standard dimensions are obtained, the MINLP is carried out once more for complete discrete decisions at the third level.

The optimization model may contain up to some ten thousands of binary 0-1 variables of alternatives. Most of them are subjected to standard and rounded dimensions. Since this number of $0-1$ variables is too high for a normal solution of the MINLP, we developed a reduction procedure, which automatically reduces binary variables for standard and rounded dimension alternatives into a reasonable number. In the optimization at the second and third level are included only those $0-1$ variables which determine standard and rounded dimension alternatives close to continuous dimensions, obtained at previous MINLP optimization iterations.

It should be noted, that the LMHS strategy can solve convex problems to global optimal solutions.

\section{Numerical examples}

The MINLP optimization approach is illustrated by two examples. The first example shows the material and standard dimension optimization of a $32 \mathrm{~m}$ long composite I beam floor system and the second one deals with the simultaneous topology, material and standard sizing optimization of an industrial steel building structure.

MINLP optimization models for the mentioned two different structures were developed. As an interface for mathematical modelling and data inputs/outputs GAMS (General Algebraic Modelling System) by Brooke et al. [16], a high level 
language, was used. The optimizations were carried out by a user-friendly version of the MINLP computer package MIPSYN, the successor of programs PROSYN by Kravanja and Grossmann [1] and TOP by Kravanja et al. [17]. The Modified OA/ER algorithm and the LMHS strategy were applied, where GAMS/CONOPT2 (Generalized reduced-gradient method), see Drud [18], was used to solve NLP subproblems and GAMS/Cplex 7.0 (Branch and Bound) [19] was used to solve MILP master problems. The structures were modelled in accordance with Eurocodes: Eurocode 4 [20] for the composite structure and Eurocode 3 [21] for steel building structure.

\subsection{Material and standard dimension optimization of a composite I beam floor system}

The first example presents the material and standard dimension optimization of a composite I beam floor system with the span of $32 \mathrm{~m}$, subjected to the selfweight and to the uniformly distributed imposed load of $7.5 \mathrm{kN} / \mathrm{m}^{2}$.

Table 1: $\quad$ Material and labour costs.

\begin{tabular}{lrl}
\hline Material costs for structural steel S 235-S 355 & $1.0-1.2$ & $\mathrm{EUR} / \mathrm{kg}$ \\
Material costs for reinforcing steel S 400 & 1.2 & $\mathrm{EUR} / \mathrm{kg}$ \\
Material costs for concrete C 25/30-C 50/60 & $100.0-120.0$ & $\mathrm{EUR} / \mathrm{m}^{3}$ \\
Sheet-iron cutting costs & 6.0 & $\mathrm{EUR} / \mathrm{m}^{1}$ \\
Cylindrical shear stud & 1.2 & $\mathrm{EUR} / \mathrm{piece}$ \\
Welding costs & 8.0 & $\mathrm{EURm}$ \\
Anti-corrosion resistant painting costs (R30) & 25.0 & $\mathrm{EUR} / \mathrm{m}^{2}$ \\
Panelling costs & 10.0 & $\mathrm{EUR} / \mathrm{m}^{2}$ \\
\hline
\end{tabular}

C $25 / 30$

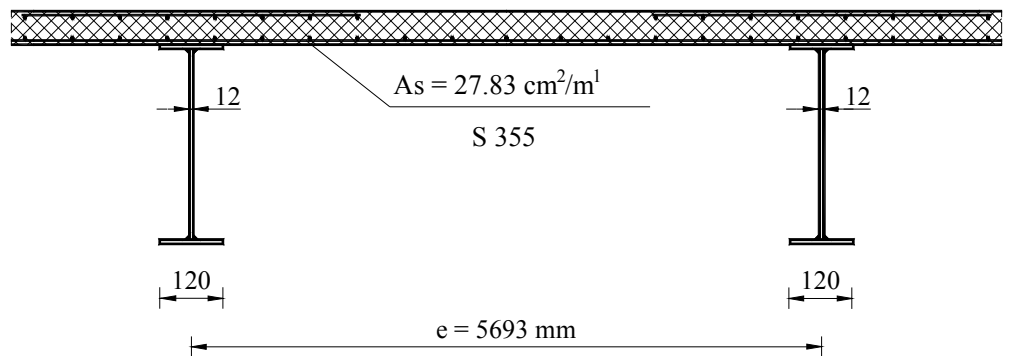

Figure 1: Optimal cross-section of the composite I-beam floor system.

The task of the MINLP optimization was to find the minimal manufacturing costs. The material and labour costs for the composite beams were thus accounted for in the economical type of the objective function, subjected to the given design, material, resistance and deflection constraints, defined according to Eurocodes 4 [20]. The material and labour costs for the composite beams considered are shown in Table 1. The superstructure comprised 6 different concrete strengths (C25, C30, C35, C40, C45, C50), 3 different structural steel 
grades (S 235, S 275, S 355), 48 various standard reinforcing steel sections as well as 9 different standard thickness of sheet-iron plates (from $8 \mathrm{~mm}$ to $40 \mathrm{~mm}$ ) for webs and flanges separately.

The optimal result of $116.90 \mathrm{EUR} / \mathrm{m}^{2}$ was obtained in the $2^{\text {nd }}$ MINLP iteration, see Figure 1. Beside the optimal self-manufacturing costs, the optimal concrete strength $\mathrm{C} 25 / 30$, steel grade $\mathrm{S} 355$, standard reinforcing wire mesh and the optimal standard thickness of webs and flanges were obtained.

\subsection{Simultaneous topology, material and standard section optimization of a single-storey industrial steel building structure}

The second example presents the topology, material and standard section optimization of a single-storey industrial steel building structure. The structure was consisted from equal non-sway steel portal frames, which are mutually connected with purlins and rails. The building was 27.5 meters wide, 72.5 meters long and 8.25 meters high. The mass of the roof was $0.20 \mathrm{~kg} / \mathrm{m}^{2}$ and of the facade cladding $0.15 \mathrm{~kg} / \mathrm{m}^{2}$. The structure was subjected to the self-weight and to the variable load. The variable imposed loads $2.00 \mathrm{kN} / \mathrm{m}^{2}$ (snow) and $0.50 \mathrm{kN} / \mathrm{m}^{2}$ (horizontal wind) were defined in the model input data.

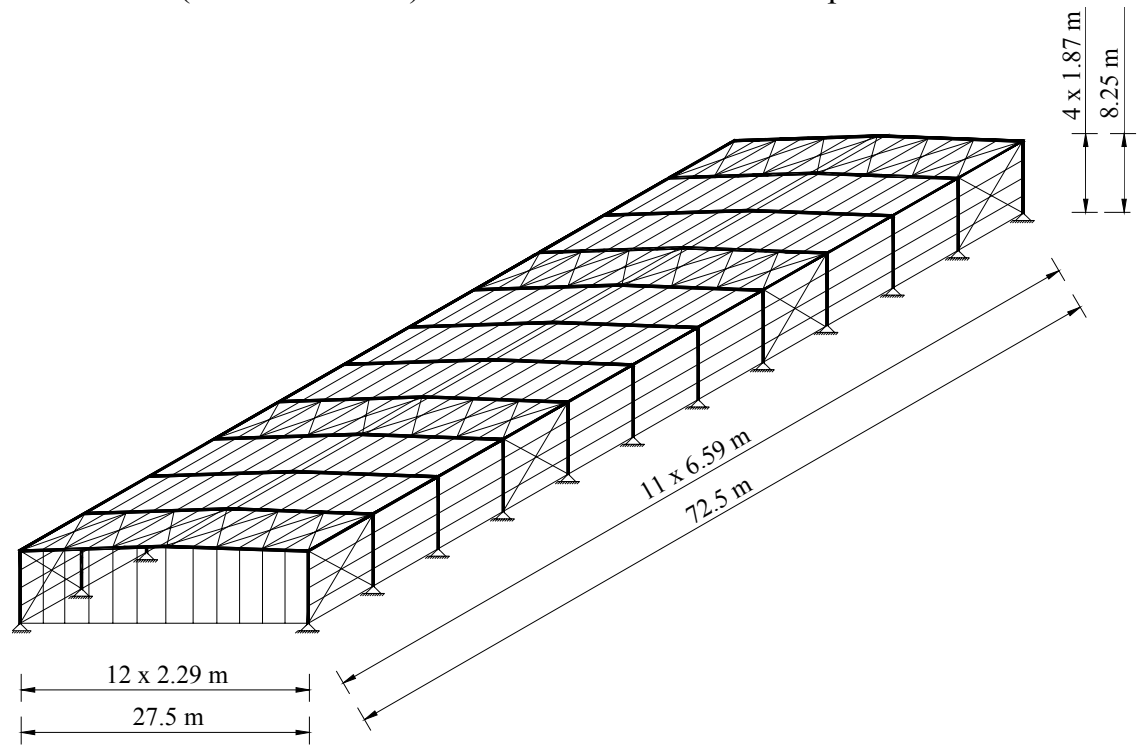

Figure 2: Optimal design of the single-storey industrial building.

The task of the performed MINLP optimization was to find the minimal structure mass. The mass objective function was thus defined for the optimization. The internal forces and deflections were calculated by the elastic first-order analysis. The design/dimensioning constraints were defined in accordance with Eurocode 3 [21]. The structure was checked for both the ultimate and serviceability limit states. The superstructure was generated in which all possible building's structures were embedded by 70 portal frame 
alternatives, 40 purlin alternatives, 20 rail alternatives, 3 discrete steel material alternatives and 24 different alternatives of standard hot rolled HEA sections (from HEA 100 to 1000) for each column, beam, purlin and rail separately.

The final optimal solution of 158.31 tons was obtained in the $4^{\text {th }}$ MINLP iteration. The optimal solution represents the mentioned "minimal" structure mass as well as the building topology of 12 portal frames, 14 purlins and 12 rails, see Figure 2. Columns and beams are both designed from HEA 700, purlins from HEA 160 and rails from HEA 100 standard sections, see Figure 3.


Figure 3: Optimal design of the portal frame.

\section{Conclusions}

The paper presents the Mixed-Integer Non-Linear Programming (MINLP) approach to structural optimization. The MINLP was found to be a successful optimization technique for solving structural optimization problems.

\section{References}

[1] Kravanja, Z. \& Grossmann, I.E., New Developments and Capabilities in PROSYN - An Automated Topology and Parameter Process Synthesizer. Computers chem. Eng., 18, pp. 1097-1114, 1994.

[2] Kravanja, S., Kravanja, Z., Bedenik, B.S., The MINLP optimization approach to structural synthesis. Part I: A general view on simultaneous topology and parameter optimization. Int. J. Numer. Methods Eng., 43, pp. 263-292, 1998.

[3] Kravanja, S., Kravanja, Z., Bedenik, B.S., The MINLP optimization approach to structural synthesis. Part II: Simultaneous topology, parameter and standard dimension optimization by the use of the Linked two-phase MINLP strategy. Int. J. Numer. Methods Eng., 43, pp. 293-328, 1998.

[4] Kravanja, S., Kravanja, Z., Bedenik, B.S., The MINLP optimization approach to structural synthesis. Part III: Synthesis of roller and sliding hydraulic steel gate structures. Int. J. Numer. Methods Eng., 43, pp. 329364, 1998. 
[5] Kravanja, S., Šilih S., Kravanja, Z., The multilevel MINLP optimization approach to structural synthesis: the simultaneous topology, material, standard and rounded dimension optimization. Internatinal Journal on Advances in Engineering Software, 36, pp. 568-583, 2005.

[6] Beale, E.M.L., Integer Programming. The State of the Art in Numerical Analysis, ed D. Jacobs, Academic Press, London, pp. 409-448, 1977.

[7] Gupta, O.K. \& Ravindran, A., Branch and bound experiments in convex nonlinear integer programming. Management Science, 31(12), pp.15331546, 1985.

[8] Olsen, G.R. \& Vanderplaats, G.N., Method for Nonlinear Optimization with Discrete Design Variables. AIAA Journal, 27(11), pp. 1584-1589, 1989.

[9] Bremicker, M., Papalambros, P.Y., Loh, H.T., Solution of Mixed-Discrete Structural Optimization Problems with a New Sequental Linearization Method. Computers \& Structures, 37(4), pp. 451-461, 1990.

[10] Westerlund, T., Pettersson, F., Grossmann, I.E., Optimization of pump configurations as a MINLP problem. Computers chem. Engng., 18(9), pp. 845-858, 1994.

[11] Benders, J.F., Partitioning Procedures for Solving Mixed-variables Programming Problems. Numerische Mathematik, 4, pp. 238-252, 1962.

[12] Geoffrion, A.M., Generalized Benders Decomposition. Journal of Optimization Theory and Applications, 10(4), pp. 237-260, 1972.

[13] Kocis, G.R. \& Grossmann, I.E., Relaxation Strategy for the Structural Optimization of Process Flowsheets. Ind. Engng Chem. Res., 26, pp. 18691880, 1987.

[14] Mawengkang, H. \& Murtagh, B.A., Solving Nonlinear Integer Programs with Large-Scale Optimization Software. Annals of Operations Research, 5, pp. 425-437, 1986.

[15] Quesada, I. \& Grossmann, I.E., An LP/NLP Based Branch and Bound Algorithm for Convex MINLP Optimization Problems. Computers Chem. Engng., 16, pp. 937-947, 1992.

[16] Brooke, A., Kendrick, D., Meeraus, A., GAMS - A User's Guide, Scientific Press, Redwood City, CA, 1988.

[17] Kravanja, S., Kravanja, Z., Bedenik, B.S., Faith, S., Simultaneous Topology and Parameter Optimization of Mechanical Structures, Proceedings of the First European Conference on Numerical Methods in Engineering (ed Ch. Hirsch et al.), Brussels, Belgium, Elsevier, Amsterdam, pp. 487-495, 1992.

[18] Drudd, A.S., CONOPT - A Large-Scale GRG Code. ORSA Journal on Computing, 6 (2), pp. 207-216, 1994.

[19] CPLEX User Notes, ILOG inc.

[20] Eurocodes 4, Design of composite structures, European Comitee for Standardization, 1992.

[21] Eurocode 3, Design of steel structures, European Committee for Standardization, 1992. 